\section{Licht + Johanniskraut = Erythem}

Johanniskrautextrakte sind zur Therapie milder Depressionen sehr beliebt. Allerdings kann es unter der Therapie zu phototoxischen Reaktionen kommen. Daher prüfte eine englische Studie, ob das Phytopharmakon die Hautreaktion auf eine hochdosierte UVA-1Bestrahlung beeinflusst.

B ritische Dermatologen verabreichten elf gesunden Probanden über zehn Tage hinweg dreimal täglich $340 \mathrm{mg}$ Johanniskrautextrakt. Dies entspricht einer Tagesdosis von $3 \mathrm{mg}$ Hypericin. Die Studienteilnehmer unterzogen sich vor und am Ende der zehntägigen Therapie zusätzlich einer UVA-1Bestrahlungsserie. Insgesamt acht Hautareale mit je $1,5 \mathrm{~cm}^{2}$ im Bereich des unteren Rückens wurden mit 7, 10, 14, 20, $28,40,56$ und $80 \mathrm{~J} / \mathrm{cm}^{2}$ bestrahlt. Vier, acht, 24, und 48 Stunden nach der Bestrahlung bestimmten die Forscher die minimale Erythemdosis (MED) und diejenige Bestrahlungsdosis, die zu einem Anstieg des Erythemindex um 0,025 geführt hatte $\left(D_{0,025}\right)$.
Die minimale Erythemdosis war nach Johanniskrautgabe zu jedem Untersuchungszeitpunkt niedriger als vor der Pharmakotherapie: Vier Stunden nach Bestrahlung betrug sie 20 vs. $28 \mathrm{~J} / \mathrm{cm}^{2}$ $(\mathrm{p}=0,11)$, acht Stunden nach Bestrahlung 14 vs. $20 \mathrm{~J} / \mathrm{cm}^{2}(\mathrm{p}=0,047)$, einen Tag nach Bestrahlung 20 vs. $40 \mathrm{~J} / \mathrm{cm}^{2}$ $(\mathrm{p}=0,055)$, zwei Tage nach Bestrahlung 28 vs. $40 \mathrm{~J} / \mathrm{cm}^{2}$ ( $\left.\mathrm{p}=0,014\right) . \mathrm{D}_{0,025}$ war bei Einnahme des Johanniskrautpräparats vier und acht Stunden nach Bestrahlung signifikant vermindert, ein und zwei Tage später war der Effekt jedoch nicht mehr signifikant.

Fazit: Eine Therapie mit Hypericin erhöht die Hautempfindlichkeit für

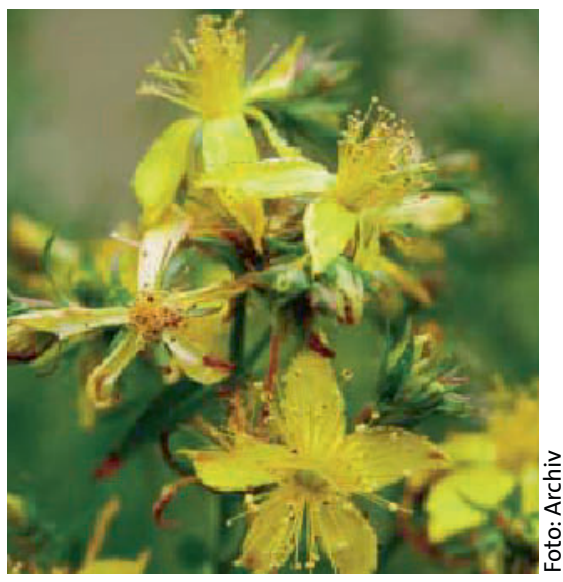

Hypericum perforatum kann unter UVATherapie phototoxisch wirken.

UVA-1. Vor einer Bestrahlungstherapie sollte daher gezielt nach einer Einnahme von Johanniskrautextrakten gefragt werden.

Beattie PE et al. Can St John's wort (hypericin) ingestion enhance the erythemal response during high-dose ultraviolet A 1 therapy? Brit J Dermatol 2005; 153: 1187-91

Eine verstärkte Apoptose von Keratinozyten wurde nicht beobachtet. Die Apoptoseinduktion von Entzündungszellen wird heute als ein Mechanismus angesehen, der eine klinisch relevante Entzündungshemmung herbeiführt.

Fazit: Im Mausmodell lindert nanokristallines Silber die Symptome einer Kontaktdermatitis genauso stark wie ein hochpotentes Glukokortikoid oder Tacrolimus. Die inflammatorischen Zytokine TNF- $\alpha$ und IL-12 werden gehemmt und die Apoptose von Entzündungszellen induziert. Damit erscheint die nanokristalline Silberzubereitung für die Therapie entzündlicher Hauterkrankungen prinzipiell als geeignet.

Bhol KC et al. Topical nanocrystalline silver cream suppresses inflammatory cytokines and induces apoptosis of inflammatory cells in a murine model of allergic contact dermatitis. Br J Dermatol 2005; 152: 1235-42 